\title{
Electromagnetic and Hadron Calorimeters in the MIPP Experiment
}

\author{
T.S. Nigmanov (presenter), H.R. Gustafson, M.J. Longo, D. Rajaram. \\ University of Michigan, Ann Arbor, MI 48109, USA
}

\begin{abstract}
The purpose of the MIPP experiment is to study the inclusive production of photons, pions, kaons, and nucleons produced in $\pi, \mathrm{K}$, and $\mathrm{p}$ interactions on various targets using beams from the Main Injector at Fermilab. The purpose of the calorimeters is to measure the production of forward-going photons and neutrons. The electromagnetic calorimeter consists of 10 lead plates interspersed with proportional chambers followed by the hadron calorimeter with 64 steel plates interspersed with scintillator. We collected data with a variety of targets with beam energies from $5 \mathrm{GeV} / \mathrm{c}$ up to $120 \mathrm{GeV} / \mathrm{c}$. The energy calibration of both calorimeters with electrons, pions, kaons and protons is discussed. The performance of the calorimeters was tested on a neutron sample.
\end{abstract}

Keywords: MIPP; Calorimeters; Showers; Calibration; Photons; Electrons; Hadrons; Fermilab PACS: $29.40 . \mathrm{Vj}$

\section{INTRODUCTION}

The MIPP (Main Injector Particle Production) experiment (E907) [1] took place in the Meson area at Fermilab. The main purposes of the experiment were to verify the scaling law of hadron fragmentation, to measure particle production from the NUMI target to predict the NUMI neutrino flux, and to measure proton-nucleus cross sections for the purpose of proton radiography. The electromagnetic and hadron calorimeters allow us to measure the production of forward-going neutral particles - photons and neutrons - since they are invisible to the upstream detectors. The electromagnetic calorimeter was built for the MIPP experiment, while the hadron calorimeter was reused from the HyperCP (E871) experiment [2].

A schematic of the MIPP setup is shown in Fig.1. The detector consisted of two magnetic spectrometers. The target was placed in front of a time projection chamber (TPC) that sits in the magnetic field of the Jolly Green Giant magnet. The TPC can identify charged particles in the low energy region $(<1 \mathrm{GeV} / \mathrm{c})$. Immediately 


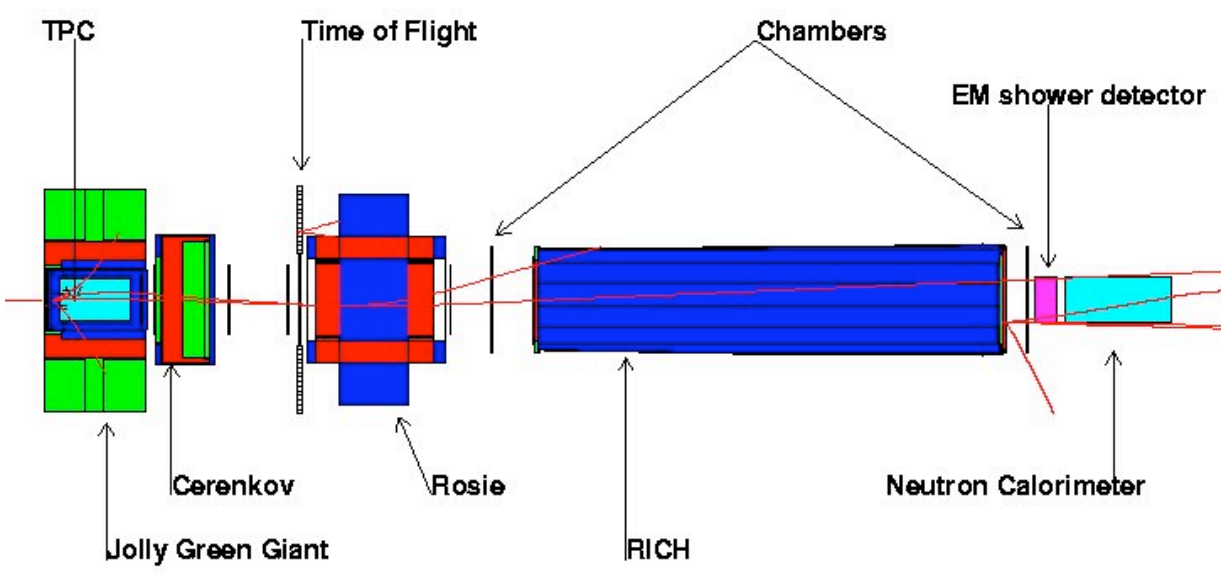

FIGURE 1. Experimental layout schematic.

downstream, a series of drift chambers and Cerenkov counters provide tracking and particle identification for the medium energy region $(1-10 \mathrm{GeV} / \mathrm{c})$. The second spectrometer consists of the Rosie dipole magnet, a time of flight detector, a series of drift and multiwire chambers, and a RICH detector, for tracking and particle identification in the high energy region. Forward-going photons and neutrons were detected by the electromagnetic and hadron calorimeters placed at the downstream end of the spectrometer. The data sample collected by MIPP is summarized in Table 1.

TABLE 1. MIPP data sample.

\begin{tabular}{lcc}
\hline Targets & Momentum $(\mathbf{G e V} / \mathbf{c})$ & ${\text { Events } \mathbf{~} \mathbf{~ 1 0}^{\mathbf{6}}}^{\mathbf{2}}$ \\
\hline LH2 & $5,20,60$ and 85 & 7.08 \\
Beryllium & 35,60 and 120 & 1.74 \\
Carbon & 20,60 and 120 & 1.33 \\
NuMI & 120 & 1.78 \\
Aluminum & 35 & 0.1 \\
Bismuth & 35,60 and 120 & 2.83 \\
Uranium & 60 & 1.18 \\
K-mass (no target) & 40 and 60 & 14.33 \\
Total & & 31.38 \\
\hline
\end{tabular}

\section{CALORIMETER SPECIFICATIONS}

A schematic of the calorimeters is shown on Fig. 2. The electromagnetic calorimeter (EMCAL) consists of 10 layers of $5.08 \mathrm{~mm}$ thick lead interspersed with planes of gas proportional chambers. The proportional chambers are made of aluminum extrusions. There are 64 anode wires with $25.4 \mathrm{~mm}$ spacing in each plane. The chambers used a gas mix of P10 (90\% Argon and $10 \%$ Methane) and $\mathrm{CF}_{4}$. The EMCAL active area was $1.6 \mathrm{~m}$ wide, $1.5 \mathrm{~m}$ high and $0.3 \mathrm{~m}$ long along the beam direction. The total thickness was $\sim 10$ radiation lengths. The EMCAL readout consisted of 640 channels. 


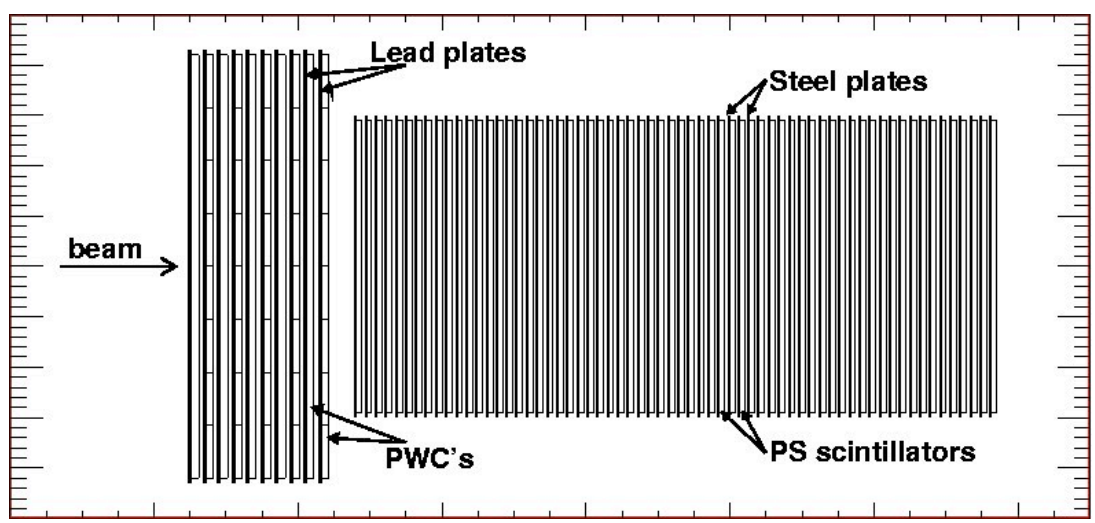

FIGURE 2. Calorimeters schematic.

The hadron calorimeter (HCAL) was composed of 64 layers of $24.1 \mathrm{~mm}$ thick Fe and $5 \mathrm{~mm}$ thick scintillator. It was originally built for the HyperCP (E871) experiment [2]. The total thickness of the HCAL was 88.5 radiation lengths (9.6 interaction lengths). Its active area was $0.99 \mathrm{~m}$ wide, $0.98 \mathrm{~m}$ high and $2.4 \mathrm{~m}$ long along the beam direction. For readout purposes it was subdivided into four longitudinal and two lateral sections, for a total of 8 cells.

\section{ENERGY CALIBRATION}

For the energy calibration of the calorimeters we assume that the response of calorimeters is a linear function of the incoming particle's energy. Then we can write

$$
E_{i}=C_{E} \sum E M C A L+C_{H} \sum H C A L
$$

where $E_{i}$ is the particle's energy measured by the upstream magnetic spectrometers, $\Sigma$ EMCAL and $\Sigma$ HCAL are summed ADC counts representing the EMCAL and HCAL responses for the passage of given particle and $C_{E}$ and $C_{H}$ are proportionality constants for the calorimeters. We selected events with a single charged track in the upstream chambers and required a match of EMCAL shower position with the projection of the incoming track. $C_{E}$ and $C_{H}$ are expected to be almost independent of energy if the ADC counts in the calorimeters are proportional to energy. The $\Sigma \mathrm{HCAL}$ vs. $\Sigma$ EMCAL dependence is illustrated in Fig. 3. In the figure on the left, the large fraction of events near $\mathrm{EMCAL}=0$ is due to protons that deposit essentially all their energy in the HCAL. However, protons deposit some energy into EMCAL planes. 

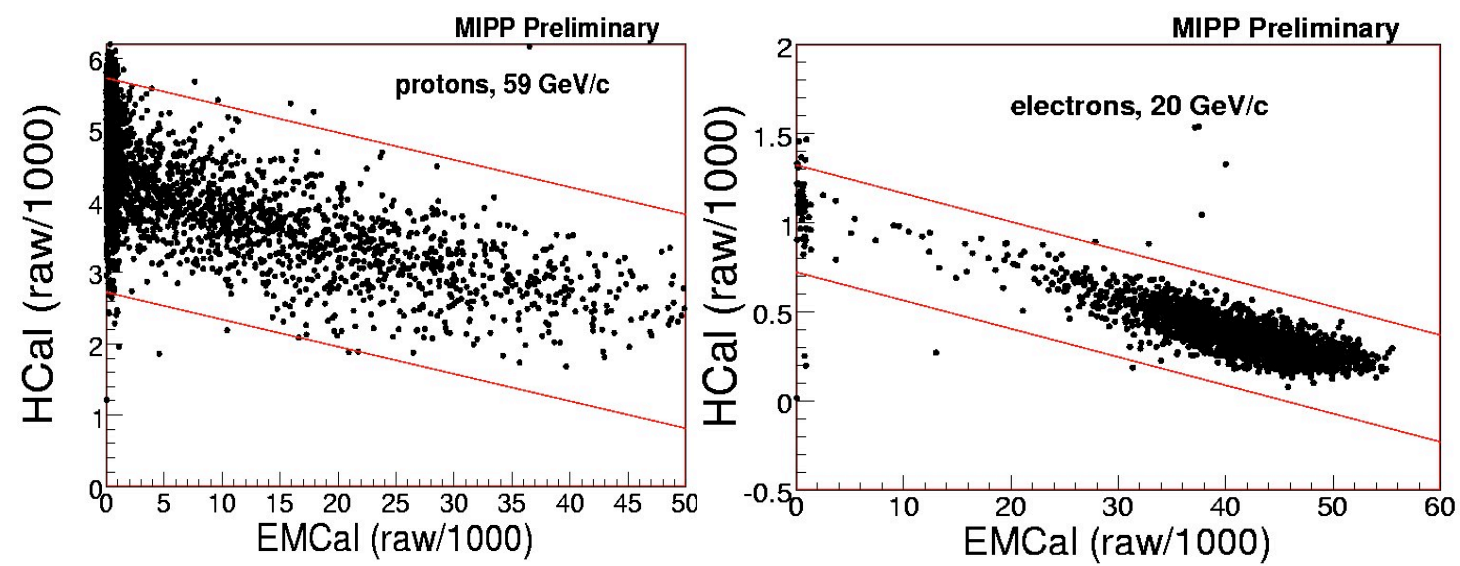

FIGURE 3. Scatter plot of HCAL vs. EMCAL responses for passage of $59 \mathrm{GeV} / \mathrm{c}$ protons (on left) and for $20 \mathrm{GeV} / \mathrm{c}$ electrons (on right). Red lines indicate the boundaries for events used in the fitting procedure.

The deposited energies in these cases lie in wide energy ranges. In contrast with protons, as we can see in the plot on the right, electrons deposit most of their energy in the EMCAL and almost nothing in the HCAL. However, there is some fraction of events near $\mathrm{EMCAL}=0$; these are presumably due to hadron contamination in the electron sample. The fit of HCAL vs. EMCAL responses is shown in Fig. 4.
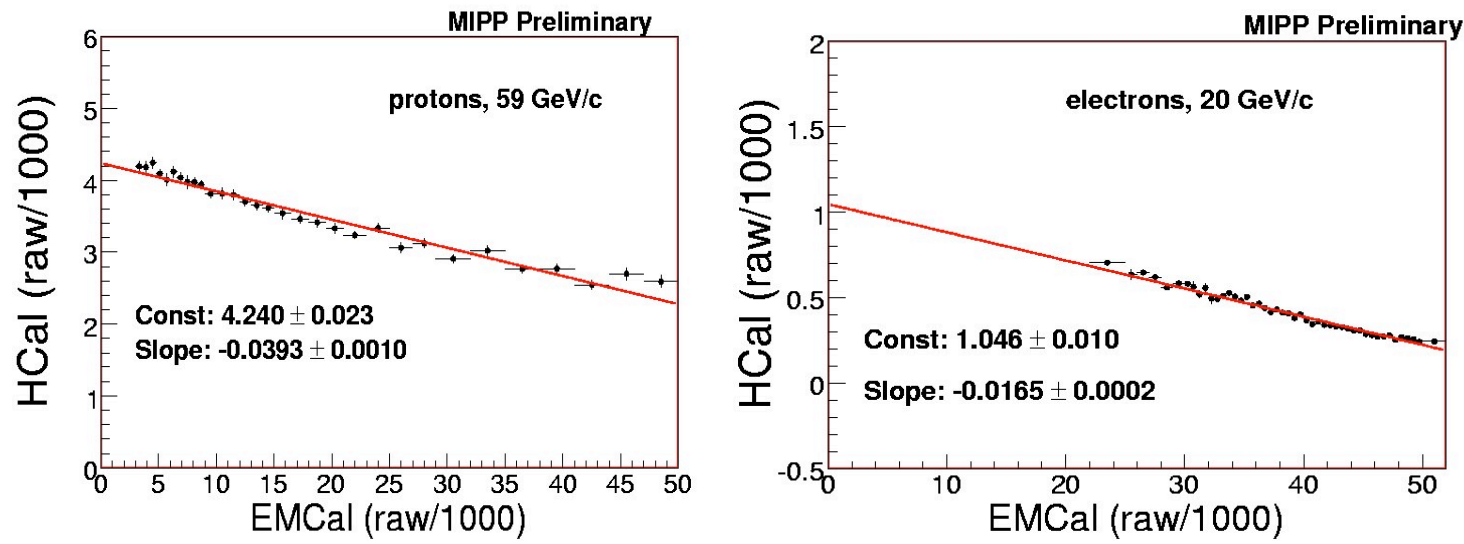

FIGURE 4. Fit of HCAL vs. EMCAL responses for $59 \mathrm{GeV} / \mathrm{c}$ protons (on left) and for $20 \mathrm{GeV} / \mathrm{c}$ electrons (on right). Red lines represent the results of linear fit.

One can see on these figures that data in both plots demonstrate first-order linear dependence. This confirms that using the fit parameters we can derive the proportionality constants. Calculations were done in two ways: by least squares fit and by a maximum likelihood method. Both methods give consistent results. The final $C_{E}$ and $C_{H}$ constants are presented in Tables 2 and 3 . The $C_{E}$ and $C_{H}$ constants in Table 2 illustrate that the EMCAL and HCAL energy responses for $\pi^{+}, \mathrm{p}$ and $\mathrm{K}^{+}$ particles are quite similar. Table 3 shows the $C_{E}$ constants derived with electrons. 
TABLE 2. The proportionality constants $C_{E}$ and $C_{H}$ for EMCAL and HCAL, respectively, calculated for $59 \mathrm{GeV} / \mathrm{c} \pi^{+}$, p and $\mathrm{K}^{+}$.

\begin{tabular}{lccc}
\hline Particles & $\mathbf{p}(\mathbf{G e V} / \mathbf{c})$ & $\boldsymbol{C}_{\boldsymbol{E}}(\mathbf{M e V})$ & $\boldsymbol{C}_{\boldsymbol{H}}(\mathbf{M e V})$ \\
\hline$\pi^{+}$ & 59 & $0.52 \pm 0.01$ & $13.2 \pm 0.1$ \\
$\mathrm{p}$ & 59 & $0.52 \pm 0.01$ & $13.9 \pm 0.1$ \\
$\mathrm{~K}^{+}$ & 59 & $0.57 \pm 0.02$ & $13.2 \pm 0.1$ \\
\hline
\end{tabular}

TABLE 3. The proportionality constants $C_{E}$ and $C_{H}$ for EMCAL and HCAL, respectively, calculated for $\mathrm{e}^{+}$and $\mathrm{e}^{-}$particles with different momenta.

\begin{tabular}{lccc}
\hline Particles & $\mathbf{p}(\mathbf{G e V} / \mathbf{c})$ & $\boldsymbol{C}_{\boldsymbol{E}}(\mathbf{M e V})$ & $\boldsymbol{C}_{\boldsymbol{H}}(\mathbf{M e V})$ \\
\hline $\mathbf{e}^{+}$ & $\sim 5$ & $0.43 \pm 0.02$ & $12.8 \pm 0.1$ \\
$\mathbf{e}^{+}$ & $\sim 10$ & $0.41 \pm 0.02$ & $12.8 \pm 0.1$ \\
$\mathrm{e}^{-}$ & 20 & $0.31 \pm 0.02$ & $13.3 \pm 0.1$ \\
\hline
\end{tabular}

Applying the $C_{E}$ and $C_{H}$ constants one can calculate the ratio of the energy deposited in the calorimeters to the momentum of incoming particle. Fig. 5 illustrates the distribution of $E_{\mathrm{e}+\mathrm{h}} / \mathrm{p}$ ratios, where $E_{\mathrm{e}+\mathrm{h}}$ is the calorimeters' response in energy units and $\mathrm{p}$ is the particle's momentum.
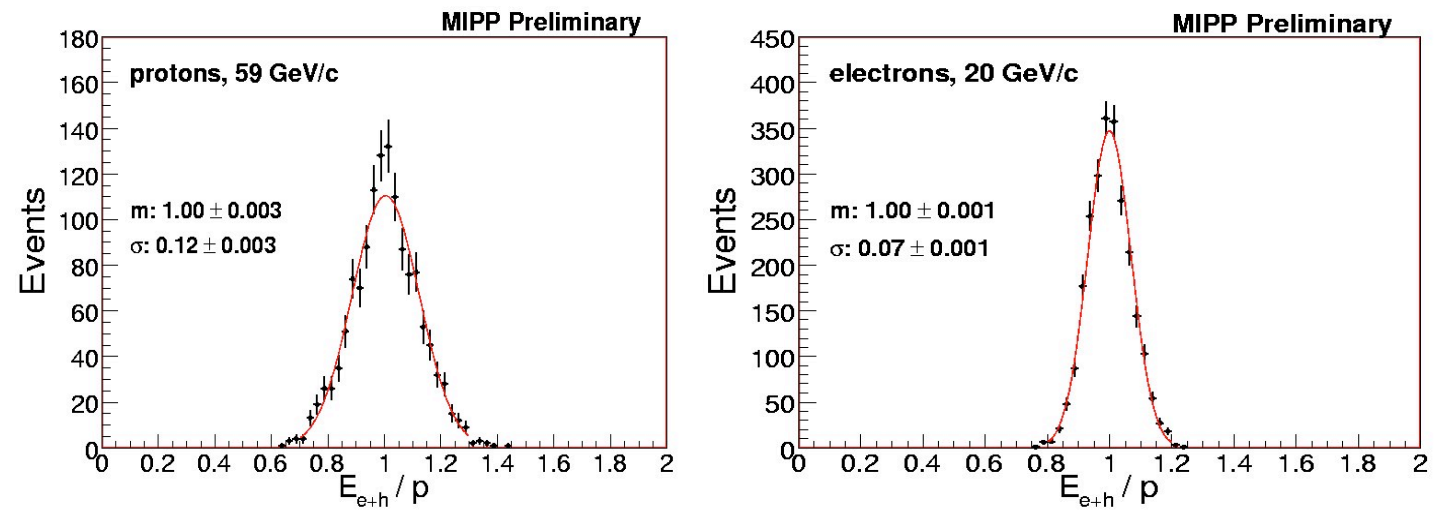

FIGURE 5. The distributions of $E_{\mathrm{e}+\mathrm{h}} / \mathrm{p}$ where $E_{\mathrm{e}+\mathrm{h}}$ is the calorimeter response in energy units, $\mathrm{p}$ is the particle's momentum. The left plot represents $59 \mathrm{GeV} / \mathrm{c}$ protons data, right plot $20 \mathrm{GeV} / \mathrm{c}$ electron data. Red curves represent the fit results using a Gaussian function.

The data in Fig. 5 shows that the mean values of $E_{\mathrm{e}+\mathrm{h}} / \mathrm{p}$ ratios are equal to 1 . This means that the calibrated calorimeter's responses and the momentum measurements by the upstream magnet spectrometers are in good agreement. The widths of the distributions reflect the energy resolution of the system.

\section{ENERGY RESOLUTION AND e/ $\pi$ RATIO}

The energy resolution of the calorimeters derived from the $E_{\mathrm{e}+\mathrm{h}} / \mathrm{p}$ widths of the distributions are presented in Table 4. One can see that the combined EMCAL and HCAL energy resolution is in good agreement with what is expected for typical sampling calorimeters [3]. Table 5 presents the $\mathrm{e} / \pi$ ratios for our calorimeters. 
TABLE 4. The combined energy resolution of both calorimeters.

\begin{tabular}{lcc}
\hline Particle & $\mathbf{p}(\mathbf{G e V} / \mathbf{c})$ & $\boldsymbol{\sigma} / \mathbf{p}(\mathbf{\%})$ \\
\hline $\mathrm{e}$ & 20 & $7 \pm 0.1$ \\
$\pi$ & 59 & $11 \pm 0.3$ \\
$\mathrm{p}$ & 59 & $12 \pm 0.3$ \\
$\mathrm{p}($ from E871) & 70 & $9(\mathrm{E} 871)$ \\
\hline
\end{tabular}

TABLE 5. The e/ $\pi$ ratios for EMCAL and HCAL calorimeters.

\begin{tabular}{lccc}
\hline Particles & $\mathbf{p}(\mathbf{G e V} / \mathbf{c})$ & e/ $\boldsymbol{\pi}(\mathbf{E M C A L})$ & $\mathrm{e} / \boldsymbol{\pi}(\mathbf{H C A L})$ \\
\hline $\mathrm{e}^{+}$ & $\sim 5$ & $0.83 \pm 0.02$ & $0.97 \pm 0.02$ \\
$\mathrm{e}^{+}$ & $\sim 10$ & $0.79 \pm 0.02$ & $0.97 \pm 0.02$ \\
$\mathrm{e}^{-}$ & 20 & $0.60 \pm 0.02$ & $1.01 \pm 0.02$ \\
\hline
\end{tabular}

As is well known, the energy response of calorimeters is significantly different for electrons (or photons) compared to hadrons because hadrons produce more energy in the form of highly ionizing tracks that are not efficiently seen by the active medium. Also some of the energy from hadrons is carried out of the calorimeter by muons and neutrinos. This effect is embodied in the famous $\mathrm{e} / \pi$ ratio. This ratio generally determines the energy resolution of hadron calorimeters because of the large fluctuations in the fraction of the energy carried off by $\pi^{0}$ 's in hadron-induced showers. For optimal energy resolution the e/ $\pi$ ratio should be 1 . In our case, with thin active layers in EMCAL, the e/ $\pi$ ratio appears to be less than 1 . However, even with the low e/ $\pi$ ratio the energy resolution appears to be quite good. This suggests that one can use a lead calorimeter with the right ratio of active to passive layer thickness and get good energy resolution. Another argument for such a design is the relatively low cost.

\section{STUDIES WITH NEUTRONS}

Another way to study the calorimeter performance is by detecting neutrons. The expected signature of neutrons in the calorimeters is: no or very low energy contribution in the EMCAL (which is about 0.5 interaction lengths) and almost all the energy deposited into the HCAL. One possible source of neutrons is the proton to neutron elastic charge exchange reaction, $p+n \rightarrow n+p$. For such neutrons we expect that the mean energy measured by the calorimeters to be consistent with the beam energy. We selected protons in the beam and used data from thin carbon and beryllium targets. For neutron selection we applied a veto on all events with any forward-going charged tracks downstream from the target. A neutron candidate event is presented in Fig. 6. 


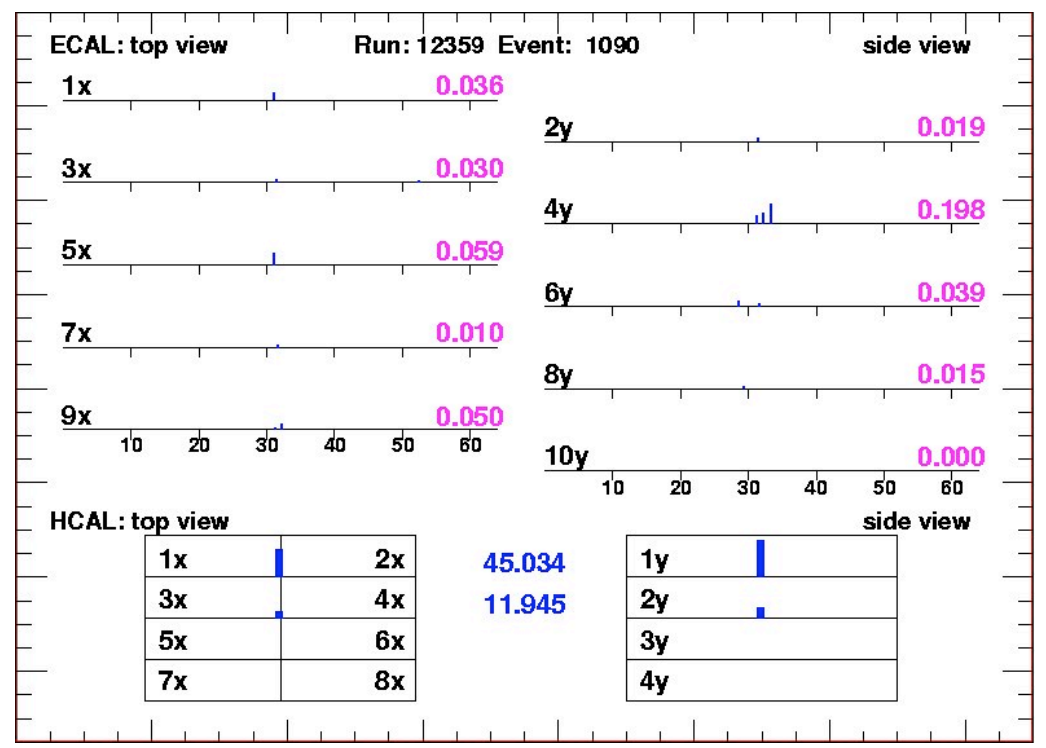

FIGURE 6. The calorimeter event display presenting a $\mathrm{p} \rightarrow$ n elastic charge exchange candidate event. The beam momentum is $59 \mathrm{GeV} / \mathrm{c}$, EMCAL energy $0.5 \mathrm{GeV}$, HCAL energy $57.0 \mathrm{GeV}$. The beam is incident from the top. Note that the HCAL is not to scale along the beam direction.

Fig. 7 represents the energy deposition of neutrons passing though the EMCAL and HCAL planes (on left plot) and the $\Sigma$ HCAL distribution (on right plot). As expected there is minimal contribution in the EMCAL planes and almost all the energy is deposited into HCAL. The mean value of the $\Sigma$ HCAL distribution (on right plot) is around $\mathrm{HCAL}=4500$ counts. This value is consistent with proton data. (See HCAL values at $\mathrm{EMCAL}=0$ on left plot in Fig. 3.) Another way to check the consistency is to compare the neutron energy with the beam energy. Fig. 8 represents the $E_{\mathrm{e}+\mathrm{h}} / \mathrm{p}$ ratios for the neutron sample. The fit results suggest that the calorimeter responses are in good agreement with the incident beam momentum.
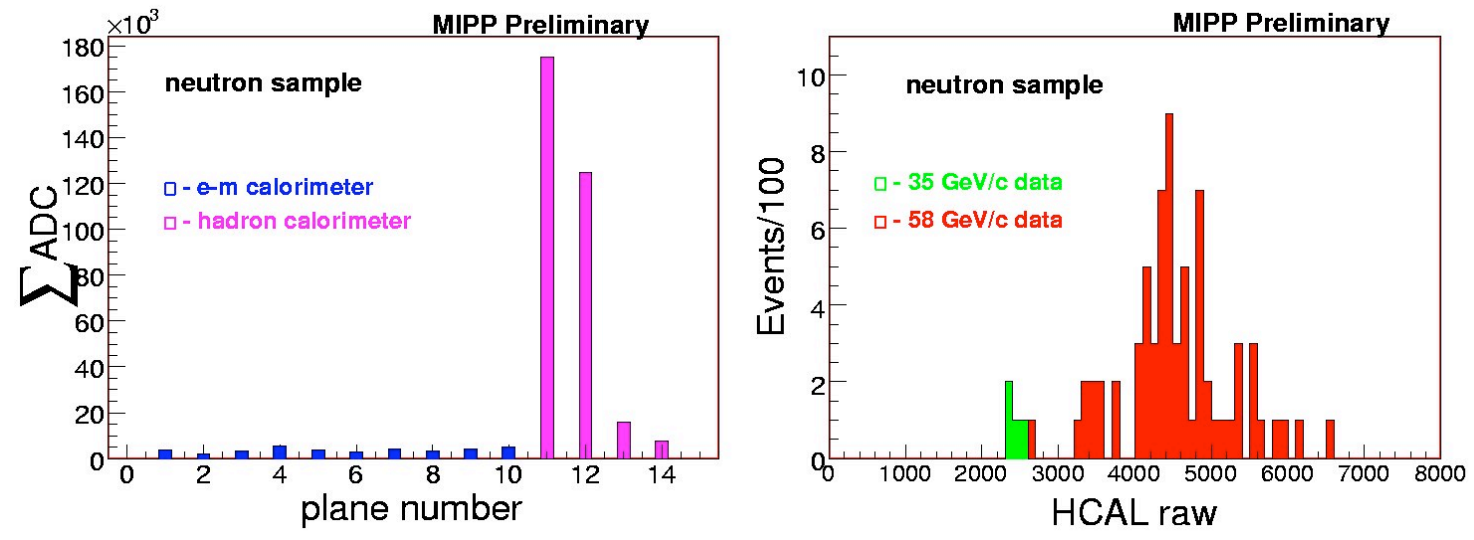


\section{MIPP Preliminary}

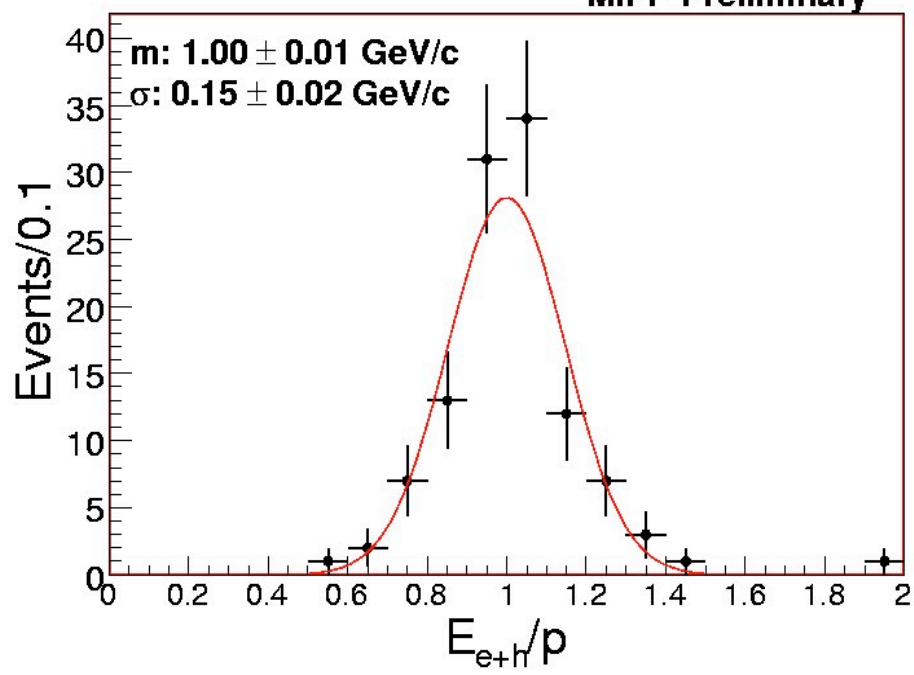

FIGURE 8. The $E_{\mathrm{e}+\mathrm{h}} / \mathrm{p}$ distribution, where $E_{\mathrm{e}+\mathrm{h}}$ is calorimeter response to neutrons, $\mathrm{p}$ is the central value of beam momentum. The red curve represents a fit with a Gaussian function.

\section{CONCLUSIONS}

The MIPP experiment collected about 31M events with hadron interactions on various targets with beam energies from 5 up to $120 \mathrm{GeV} / \mathrm{c}$. Charged particles were identified in a wide range of momenta. Use of the electromagnetic and hadron calorimeters made it possible to detect photons and neutrons.

The calorimeters were energy calibrated using $\mathrm{e}^{+}, \mathrm{e}^{-}$and hadrons. The EMCAL and HCAL data demonstrate a linear response with respect to the incoming particle's energy. The calibration constants give an e/ $\pi$ ratio less than 1 . However the measured energy resolution was found to be quite good, and it is consistent with what was expected for the sampling calorimeters $(\sigma / \mathrm{E} \sim 10 \%)[3]$.

The calorimeter performance was tested on a neutron sample. It demonstrates adequate response for neutrons: very low energy deposition in EMCAL and all the energy deposited into HCAL. The mean value of neutron energy was found to be consistent with the beam energy.

\section{ACKNOWLEDGMENTS}

The authors express their thanks to colleagues on the MIPP experiment for their efforts in running the experiment and data processing. We thank the committee for the pleasant atmosphere in the conference. This research was sponsored by the National Nuclear Security Administration under the Stewardship Science Academic Alliances program through DOE Research Grant DE-FG52-2006NA26182.

\section{REFERENCES}

1. P. D. Barnes Jr., et al. Proposal to measure particle production in the Meson area using Main Injector primary and secondary beams. http://ppd.fnal.gov/experiments/E907/E907.html

2. R. A. Burnstein, et al., Nucl. Instrum. and Methods 541, 516 (2005).

3. Review of Particle Physics. Physics Letters B 592 (2004) 265. 